\title{
Impact of Stent-Assisted Recanalization of Carotid Artery Stenosis on Brain Volume Changes
}

\author{
Ömer Fatih Nas ${ }^{1}$, Aylin Bican Demir ${ }^{2}$, Mustafa Bakar², Güven Özkaya ${ }^{3}$, Emre Kaçar ${ }^{4}$, Bahattin \\ Hakyemez \\ ${ }^{1}$ Department of Radiodiagnostics, Uludağ University, School of Medicine, Bursa, Türkiye \\ ${ }^{2}$ Department of Neurology, Uludağ University, School of Medicine, Bursa, Türkiye \\ ${ }^{3}$ Department of Biostatistics, Uludağ University, School of Medicine, Bursa, Türkiye \\ ${ }^{4}$ Department of Radiodiagnostics, Afyon Kocatepe University, School of Medicine, Afyonkarahisar, Türkiye
}

\begin{abstract}
Objective: The purpose of this study was to investigate the effect of recanalization on stenotic internal carotid artery on brain volume changes in stent applied patients.
\end{abstract}

Materials and Methods: Carotid stenting was performed in 17 patients with severe carotid artery stenosis between June 2013 and April 2014. High resolution 3D T1 weight images were obtained from each patient 24 hours before and $7.2 \pm 3.6$ months (mean \pm standard deviation) after the procedure on a $3 \mathrm{~T}$ magnetic resonance imaging scanner. Intracranial total cortical grey matter, total cortical white matter, white matter hypointensity, total intraventricular and subcortical grey matter volumes were assessed by FreeSurfer version 4.5.0.

Results: A significant reduction was found in total cortical white matter and subcortical grey matter volumes $(p<0.05)$. A significant increase was found in white matter hypointensity and total intraventricular volumes after procedure $(p<0.05)$. However, no statistical significant difference was found in total cortical grey matter volume before and after procedure $(p=0.902)$.

Conclusion: The significant reduction in the postoperative intracranial total cortical white matter and subcortical grey matter volumes and the significant increase in the white matter hypointensity volume were considered to be secondary to neuronal damage. J Clin Exp Invest 2016; 7(4): 283-289

Key words: Brain volume changes, carotid stenosis; magnetic resonance imaging

\section{INTRODUCTION}

Patients with stenosis of internal carotid artery (ICA) have an annual stroke risk of $5-6 \%$ which increases to $70 \%$ if more stenosis occurs. Carotid endarterectomy (CEA) and carotid artery stenting (CAS) are the methods performed to avoid the potential risk of stroke [1].

In severe stenosis, cerebral perfusion might be reduced due to decreased blood supply. To diagnose cerebral microcirculationin patients with stenosis of ICA, several imaging techniques such as cervical and transcranial doppler, perfusion computed tomography (CT), intracarotid xenonium injection and perfusion magnetic resonance imaging (MRI) are needed. However, post-CAS hemodynamic changes in cerebral perfusion are not yet clearly known [2].

MR image-guided surface or voxel-based brain analysis methods help to assess cerebral cortex thickness. FreeSurfer is one of the surfacebased methods, which is most commonly used and is a frequently referred methodology in clinical trials [3]. It is an easy-to-use brain analysis software which provides accurate and automated image analysis [4]. To our knowledge, there are no published studies that investigated the effect of stent-assisted 
recanalization on brain volume changes by using the software FreeSurfer.

In this study, we investigated the effect of recanalization on stenotic ICA on brain volume changes in stents applied patients by evaluating T1-weighted 3D MR images before and after the procedure.

\section{METHODS}

\section{Patients}

Carotid stenting was performed in 17 patients ( 9 on the left, 4 on the right side and 4 on both sides, 21 total stents) with severe ICA stenosis between June 2013 and April 2014. Severe stenosis is defined by the North American Symptomatic Carotid Endarterectomy Trial (NASCET) as more $>70 \%$ narrowing of the vessel [5]. Inclusion criteria were as follow; a history of carotid stenting with severe (more than $70 \%$ ) carotid stenosis, a T1-weighted 3D MRIs before and after the procedure and cerebral ischemic risk. Patients not meeting these criteria were excluded. Fourteen males (82.4\%) and 3 female were enrolled $(17.6 \%)$. The mean degree of stenosis was $89.4 \pm 7.8 \%$ on the right side and $86.5 \pm 7.5 \%$ on left side. The patients' mean age was $65.8 \pm 5.7$ years (age range $56-77$ years). Table 1 shows the co-morbidities of all patients i.e. $88 \%$ of the patients had hypertension $(n=15)$, $47 \%$ had coronary artery disease $(n=8), 41 \%$ had smoking history $(n=7), 18 \%$ had diabetes $(n=3), 18 \%$ had a history of cerebrovascular event $(n=3)$, and $12 \%$ hyperlipidemia $(n=2)$. High resolution 3D T1-weighted images were acquired on a 3T MRI scanner using 32-channel head coil 24 hours before and an average of 7.18 \pm 3.6 months after the procedure (ranging from 2 to 12 months). Intracranial total cortical grey matter, total cortical white matter, white matter hypointensity, total intraventricular and subcortical grey matter volumes were assessed by using the software FreeSurfer version 4.5.0. Approval was obtained from the ethics committee before the study.

\section{Interventional Technique}

Informed consent was obtained from all patients that were scheduled for endovascular stent placement. Acetylsalicylic acid (Aspirin $\circledast$; Bayer) 100-300 mg/day and clopidogrel (Plavix®;
Sanofi-Synthalebo) 75 mg/day p.o. was administered at least 72 hours prior to the procedure. Stenting was performed under sterile conditions and local anesthesia ( $2 \%$ prilocaine; Citanest, AstraZeneca) using a biplane, flatpanel angiography unit (AXIOM Artis FD Biplane Angiosuite; Siemens Medical Solutions). An 8F introducer was inserted into the right common femoral artery using the single-wall puncture technique. During the procedure, 5000-7000 units of heparin were administered intraarterially. Either right or left common carotid artery (CCA) was reached by using a guidewire (Roadrunner; Cook) and an 8F guiding catheter (Envoy; Cordis) depending on the stenosis site. Right or left ICA stenosis was determined on the angiographic images. The stenosis was crossed with a filter device (Emboshield NAV ${ }^{6}$; Abbott vascular). The filter was opened in the petrous portion of the ICA. After opening the filter, a $3 \times 20 \mathrm{~mm}$ balloon (Empira; Cordis) was used for pre-dilatation. Then, a self-expanding nitinolcarotid stent [Protege stent $(n=9)$; Covidien, Xact stent $(n=7)$; Abbott vascular and Precise stent $(n=5)$; Cordis] was placed into the ocluded segment. ICA reconstruction was obtained with $5 \times 20 \mathrm{~mm}$ balloons (Viatrac 14 Plus; Abbott vascular and Aviator Plus; Cordis) after the stenting. The filter was removed from the petrous portion of the ICA and the femoral artery access site was closed with a vascular closure system (StarClose; Abbott vascular). Immediately after the procedure, $1000 \mathrm{U} /$ hour intravenous heparin was administrated for 8 hours, followed by enoxaparine (Clexane $\AA$, Aventis) $0.6 \mathrm{ml} /$ day s.c. for three days. No clinical deficit occurred in the patients following the successful endovascular treatment. Stable patients were discharged one day after operation and prescribed acetylsalicylic acid $100-300 \mathrm{mg} /$ day for lifelong and clopidogrel 75 $\mathrm{mg} /$ day for 3-6 months.

\section{Magnetic Resonance Imaging}

Conventional axial, sagittal and coronal $\mathrm{T} 1$ and T2-weighted images were acquired on a $3 T$ scanner (Achieva 3.0T Tx; Philips) using a 32channel head coil. High resolution T1-weighted 3D images were acquired using 3D-FFE (three dimensional fast field echo) sequence with scan time of approximately 5.5 minutes. The parameters were as followed: repetition time (TR)/echo time (TE): 8.2/3.8, inversion time (TI): 
$1018 \mathrm{~ms}$, number of signal averages (NSA): 1 , flip angle: $8^{\circ}$, field of view (FOV): $240 \times 240 \mathrm{~mm}$, matrix: $240 \times 240$, slice voxel size: isovolumetric $1 \mathrm{~mm}$, and 170 slices.

\section{Morphometric Analyses}

Images acquired by the high resolution MRI were transferred into a DICOM format for Macintosh-based computer. FreeSurfer4.5.0 software was used for morphometric analyses. First, distortions on the images caused by the patient movements and variations of brightness caused by the changes in the B1 field were corrected. Second, images were placed on the Talairach coordinate system which allowed for pre-labeling by using manually created standard brain templates. This led to the increased success of segmentation. Also errors caused by pathologies were reduced this way. Tabula was automatically deleted, and the rest was used as a brain mask for labeling and segmentation.

Outcomes of automatic deletion and segmentation were reviewed. If necessary; corrected and volume measurements were repeated by a specialist radiologist. Postprocessing took approximately 8 hours in each patient. This way, measurements of total cortical grey matter, total cortical white matter, white matter hypointensity, total intraventricular and subcortical grey matter volumes were obtained and used in statistical analysis.

\section{Statistical analyses}

All statistical analyses were performed with SPSS 22.0 statistical program. Whether data demonstrated a normal distribution or not was determined using Shapiro-Wilk test. Descriptive values of variables were expressed as means, standard deviations, medians, minimums and maximums. Wilcoxon signed rank test and matched pairs t-test were used for group comparison. The level of significance was determined as 0.05 .

\section{RESULTS}

After CAS, no statistical significant difference was observed in the total cortical grey matter volumes $(p=0.902)$. The mean total cortical grey matter volume was $381875 \pm 41334 \mathrm{~mm}^{3}$ before and $381496 \pm 41757 \mathrm{~mm}^{3}$ after CAS. On the other hand, a significant reduction was observed in the total cortical white matter and subcortical grey matter volumes $(p<0.05)$. The mean total cortical white matter and subcortical grey matter volumes were $428110 \pm 75521 \mathrm{~mm}^{3}$ and 157333 $\pm 18077 \mathrm{~mm}^{3}$ before and $412200 \pm 73801 \mathrm{~mm}^{3}$ and $155154 \pm 17297 \mathrm{~mm}^{3}$ after CAS, respectively.

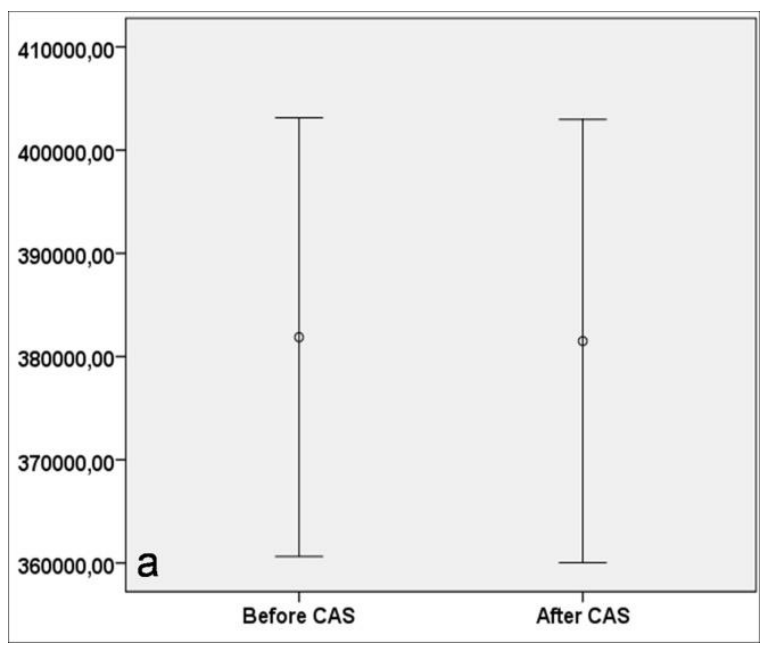

Figure 1. Graphical analyses of pre and post-CAS cerebral volumes: Total cortical grey matter volume.

A significant increase was observed in the total intraventricular and white matter hypointensity volumes $(p<0.05)$. The median total intraventricular and white matter hypointensity volumes were (min-max) $30945 \mathrm{~mm}^{3}$ (15944$57931)$ and $4591 \mathrm{~mm}^{3}$ (1980-20464) before and $34967 \mathrm{~mm}^{3}$ (19333-76057) and $6491 \mathrm{~mm}^{3}$ (2571-22526) after CAS, respectively (Figures 15 , Table 2).

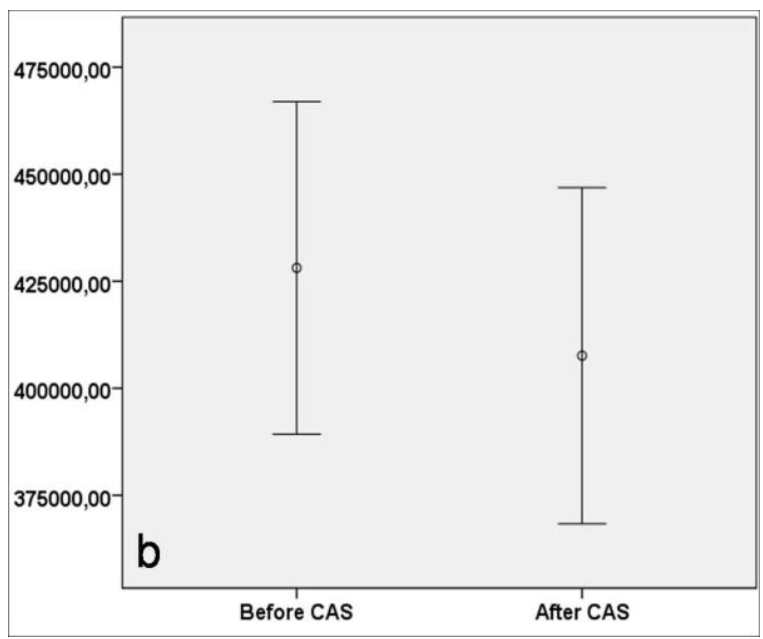


Figure 2. Graphical analyses of pre and post-CAS cerebral volumes: Total cortical white matter volume.

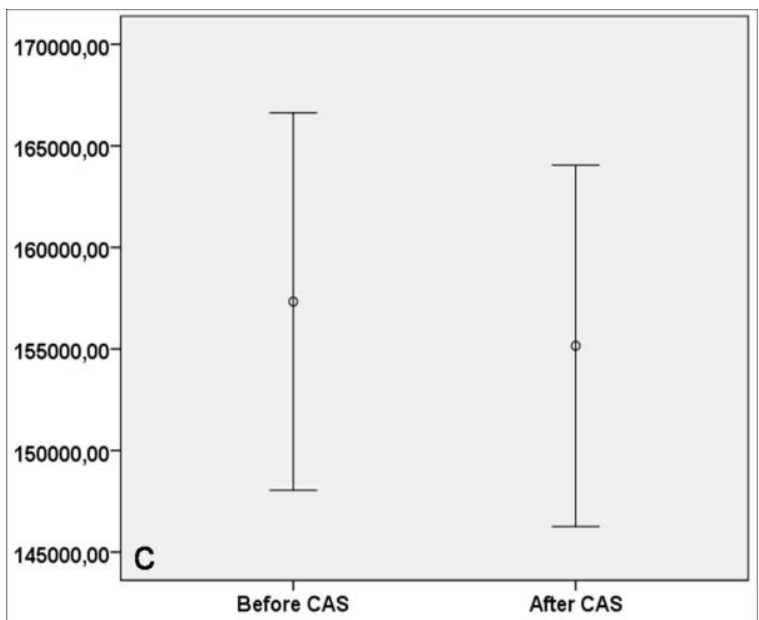

Figure 3. Graphical analyses of pre and post-CAS cerebral volumes: Subcortical grey matter volume.

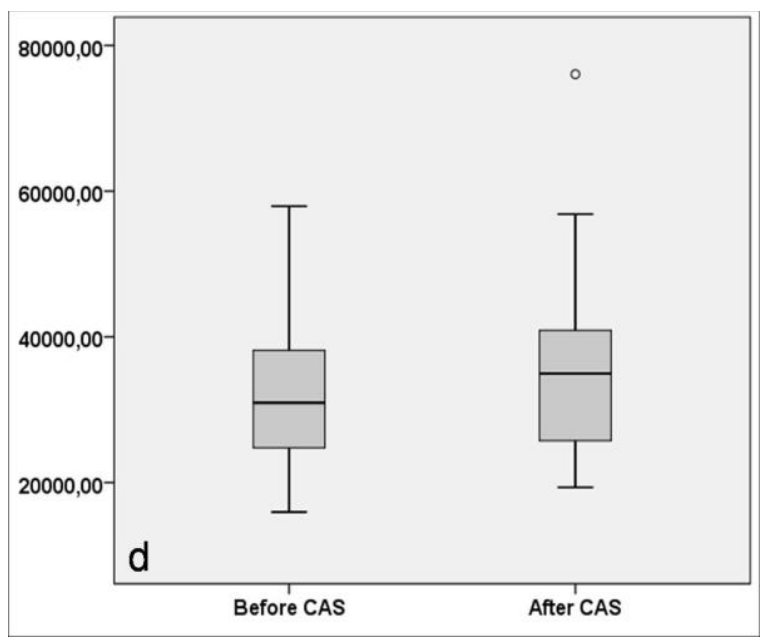

Figure 4: Graphical analyses of pre and post-CAS cerebral volumes: Total intraventricular volume.

Table 1. Demographic data of 17 patients.

\begin{tabular}{|c|c|c|c|c|c|c|c|c|}
\hline $\begin{array}{l}\text { Patients' } \\
\text { number }\end{array}$ & $\begin{array}{l}\text { Sex/ } \\
\text { age }\end{array}$ & $\begin{array}{c}\text { Carotid stenosis (side and degree } \\
(\%))\end{array}$ & HT & CAD & $\begin{array}{l}\text { History of } \\
\text { smoking }\end{array}$ & $\begin{array}{l}\text { History of } \\
\text { CVE }\end{array}$ & DM & HL \\
\hline 1 & $\mathrm{M} / 69$ & L: 95 & + & + & + & - & - & - \\
\hline 2 & $\mathrm{~F} / 65$ & $\mathrm{R}: 95, \mathrm{~L}: 75$ & + & - & - & - & - & + \\
\hline 3 & $\mathrm{M} / 63$ & $\mathrm{~L}: 90$ & + & - & + & - & + & - \\
\hline 4 & $\mathrm{M} / 71$ & $L: 90$ & + & - & - & - & - & + \\
\hline 5 & $\mathrm{M} / 56$ & R:75, L: 80 & + & - & + & - & - & - \\
\hline 6 & $\mathrm{M} / 63$ & $L: 90$ & + & - & - & + & - & - \\
\hline 7 & $\mathrm{M} / 64$ & $\mathrm{R}: 95$ & + & - & + & - & - & - \\
\hline 8 & M/64 & R:95, L: 75 & + & + & - & - & + & - \\
\hline 9 & $\mathrm{M} / 60$ & $\mathrm{R}: 95$ & - & + & + & - & - & - \\
\hline 10 & $\mathrm{M} / 61$ & $\mathrm{R}: 90$ & + & - & - & + & - & - \\
\hline 11 & $\mathrm{~F} / 74$ & R:90, L: 95 & + & + & - & - & - & - \\
\hline 12 & $\mathrm{M} / 64$ & $\mathrm{~L}: 80$ & + & + & + & - & - & - \\
\hline 13 & $\mathrm{M} / 69$ & $\mathrm{R}: 80$ & + & + & - & - & - & - \\
\hline 14 & $\mathrm{~F} / 77$ & $\mathrm{~L}: 90$ & + & + & - & + & - & - \\
\hline 15 & $\mathrm{M} / 61$ & L: 95 & + & + & - & - & - & - \\
\hline 16 & $\mathrm{M} / 74$ & L: 90 & - & - & + & - & - & - \\
\hline 17 & $\mathrm{M} / 63$ & $\mathrm{~L}: 80$ & + & - & - & - & + & - \\
\hline
\end{tabular}

M: Male, F: Female, R: Rıght, L: Left, HT: Hypertension, CAD: Coronary Artery Disease, CVE: Cerebrovascular Events, DM: Diabetes Mellitus, HL: Hyperlipidemia

Table 2. Statistical analyses of pre and post-CAS cerebral volumes

\begin{tabular}{|c|c|c|c|}
\hline Parameters & Before CAS ${ }^{\star}$ & After CAS* & p \\
\hline Total cortical grey matter $\left(\mathrm{mm}^{3}\right)$ mean $\pm \mathrm{sd}$ & $381875 \pm 41334$ & $381496 \pm 41757$ & 0.902 \\
\hline Total cortical white matter $\left(\mathrm{mm}^{3}\right)$ mean \pm sd & $428110 \pm 75521$ & $412200 \pm 73801$ & 0.005 \\
\hline Subcortical grey matter $\left(\mathrm{mm}^{3}\right)$ mean \pm sd & $157333 \pm 18077$ & $155154 \pm 17297$ & 0.016 \\
\hline $\begin{array}{l}\text { Total intraventricular }\left(\mathrm{mm}^{3}\right) \text { Median (min - } \\
\text { max) }\end{array}$ & $30945(15944-57931)$ & 34967 (19333 - 76057) & 0.009 \\
\hline $\begin{array}{l}\text { White matter hypointensity }\left(\mathrm{mm}^{3}\right) \text { Median } \\
\text { (min - max) }\end{array}$ & $4591(1980-20464)$ & $6491(2571-22526)$ & 0.017 \\
\hline
\end{tabular}




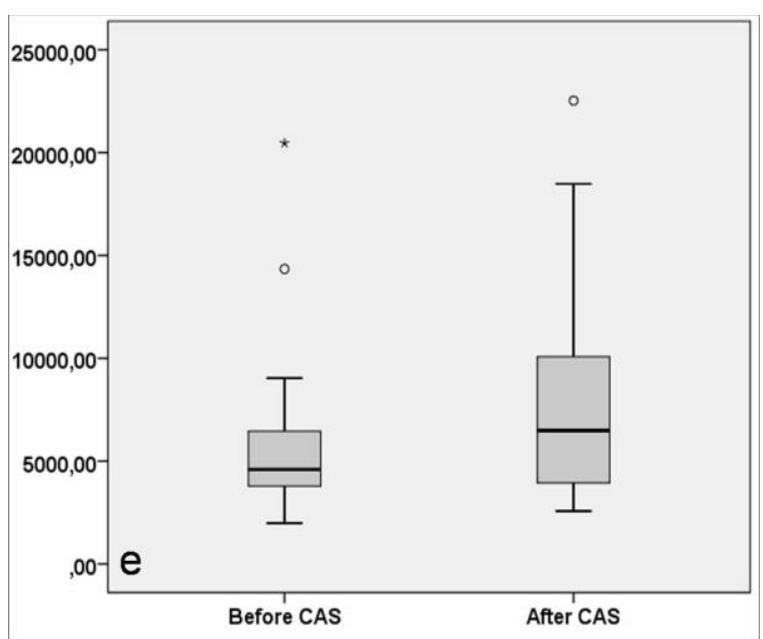

Figure 5. Graphical analyses of pre and post-CAS cerebral volumes: Total intraventricular volume, (e) White matter hypointensity volume.

\section{DISCUSSION}

Magnetic resonance angiography (MRA) and transcranial doppler demonstrated that collateral circulation in patients with ICA stenosis did not played a role in assessment of the brain perfusion. Digital subtraction angiography (DSA) also provided significant information about the collateral circulation. Positron emission tomography (PET) and single photon emission computed tomography (SPECT) are invasive methods, whereas arterial spin-labeling (ASL) $M R I$ is a non-invasive method for the assessment of regional cerebral blood flow (CBF) [6]. Van Laar et al [6] observed a 15\% increase in post-CAS regional CBF levels in the ipsilateral hemisphere whereas and Ko et al [7] observed a $21 \%$ increase when using ASL-MRI and SPECT.

Distal perfusion pressure and CBF are normal when the collateral pathways are sufficient in severe carotid stenosis [8,9]. If the collateral pathways are insufficient and the perfusion pressure distal to the stenosis is reduced, then autoregulatory dilation can keep the CBF at normal levels. When autoregulatory dilation capacity is exceeded, the CBF will be reduced relative to the cerebral rate of oxygen metabolism (CMRO2). In addition, oxygen extraction fraction (OEF) will be increased to maintain the normal CMRO2. This condition is called 'misery perfusion' or 'stage II hemodynamic failure', and is the most severe stage of hemodynamic impairment $[8,10]$. Cerebral vascularization and the brain may adapt to the chronic reduction in CBF in areas of the brain with no cerebral infarction through several potential mechanisms. While CBF may increase with the development of collateral ways, CBF can decrease in order to provide the balance of $\mathrm{CMRO}$ 2. It is suggested that reduced CMRO2 in normal brain areas can cause selective ischemic neuronal loss [8]. Based on this study, we believe that the chronic hypoxia in patients with stenotic ICA leads to ischemic neuronal loss.

Adult human brain accounts for $2 \%$ of the total body weight, receives $15 \%$ of the total cardiac output and consumes $20 \%$ of the inhaled oxygen. The blood flow delivers oxygen and glucose to neurons needed for transmembrane ion transport, electrical activities, synaptic transmission, macromolecular synthesis, intracellular transport and cytoskeletal integrity. A normal CBF rate is $50-60 \mathrm{~mL} / 100 \mathrm{~g} / \mathrm{min}$ in primates and $100 \mathrm{~mL} / 100 \mathrm{~g} / \mathrm{min}$ in rats and gerbils. A $50 \%$ reduction in $\mathrm{CBF}$ can be tolerated. However, a reduction between 25$50 \%$ in CBF can lead to ischemic injury due to inhibition of protein synthesis, prevention of the flow of transient potassium and calcium ions, cytotoxic edema and acidosis. This injury may be followed by neuronal apoptosis. A CBF less than $25 \%$ leads to rapidly loss of neuron functions whereas a CBF less than $15-20 \%$ of normal values leads to irreversible neuronal damage. The brain is quite susceptible to both focal and global ischemia. Unless ischemia is reversed, reperfusion increases the ischemic injury. Excitotoxicity, impaired calcium ion homeostasis, nitric oxide, free radicals, inflammation and apoptosis can be responsible for cerebral ischemia and reperfusion injury. Nitric oxide and free radicals may either do direct damage or cause indirect damage by means of inflammation and apoptosis [11].

Atherosclerotic risk factors like hyperlipidemia, diabetes, hypertension, smoking and aging may induce release of reactive oxygen species from endothelium, vascular smooth muscle cells and adventitial cells which can lead to impair cerebral autoregulation. Hypoxia caused by 
cerebral ischemia may affect a number of neurons at different levels. Several factors such as hypoxia and cerebral ischemia are known to induce neuronal apoptosis in the nervous system [12]. Chen et al [13] demonstrated nuclear DNA damage after middle cerebral artery stenosis and reperfusion in rats, which was indicative of neuronal apoptosis. Pulsinelli et al [14] investigated ischemic neuronal damage following transient bilateral forebrain ischemia in a Wistar rat model of four-vessel stenosis. They occluded vertebral arteries permanently and 24 hours later common carotid arteries transiently for 10,20 and 30 minutes. After 10 minutes of stenosis, ischemic cell changes were observed in cerebral hemispheres of the rats, and after 30 minutes of stenosis early neuronal damage occurred. The authors concluded that neuronal damage progressively advanced with time.

MR image-guided cerebral cortex thickness estimation methods can be categorized into two groups: surface and voxel-based brain analysis methods. Both methods require initial segmentation of grey matter, white matter and cerebrospinal fluid. Laplacian and Registration are voxel-based methods whereas the FreeSurfer is a surface-based method. Surfacebased methods are more widely used compared to voxel-based ones due to accessible software packages such as BrainSuite, BrainVISA and FreeSurfer. The FreeSurfer is the most common method employed in clinical trials beyond all surface-based methods [3], because it is easy to use and rapidly developing brain analysis software for the assessment of functional and structural features and connections in the brain. FreeSurfer is a freeware and can be improved with hardware and software platforms. It allows cross-modal intra-subject registration, combined volume and surface cross-subject registration, probabilistic estimation of cytoarchitectonic boundaries, automated tractography and longitudinal analysis. Furthermore, the software can also be used for the assessment of neurological and genetic basis of neuroanatomical disorders, healthy development and aging [4]. Liem et al [15] reported that surface-based metric parameters (cortical thickness, surface area and volume) of the FreeSurfer have high reliability in the assessment of age-related structural alterations in brain regions of healthy elderly individuals. In our study, we preferred the FreeSurfer software since its easy-to-use nature and the accurate and automate image analysis properties.

This study had some limitations. First, the number of patients included in the study was limited. Second, no CEA control group was analyzed. Third, only the FreeSurfer surfacebased method was used. Forth, no clinical examination was performed after CAS. Last, this was a retrospective study without a control group.

In conclusions, intracranial neuronal loss in patients treated with CAS for severe ICA stenosis might be due to neuronal damage as determined by FreeSurfer surface-based volumetric measurements. Furthermore, the significant increase in the total intraventricular volume might be related to the reduction in the total white matter volume. Evaluation of this study together with postmortem studies and other brain analysis softwares will be useful.

Declaration of conflicting interests: The authors declared no conflicts of interest with respect to the authorship and/or authorship of this article.

Funding: The authors received no financial support for the research and/or authorship of this article.

\section{REFERENCES}

1. Yun TJ, Sohn $\mathrm{CH}$, Han $\mathrm{MH}$, et al. Effect of carotid artery stenting on cerebral blood flow: evaluation of hemodynamic changes using arterial spin labeling. Neuroradiology 2013;55:271-81.

2. Tavares A, Caldas JG, Castro CC, et al. Changes in perfusion-weighted magnetic resonance imaging after carotid angioplasty with stent. Interv Neuroradiol 2010;16:161-69.

3. Clarkson MJ, Cardoso MJ, Ridgway GR, et al. A comparison of voxel and surface based cortical thickness Estimation methods. Neuroimage 2011;57:856-65.

4. Fischl B. FreeSurfer. Neuroimage. 2012;62:774-81.

5. Fox AJ. How to measure carotid stenosis. Radiology 1993;186:316-18. 
6. Van Laar PJ, Hendrikse J, Mali WP, et al. Altered flow territories after carotid stenting and carotid endarterectomy. J Vasc Surg 2007;45:1155-61.

7. Ko NU, Achrol AS, Chopra M, et al. Cerebral blood flow changes after endovascular treatment of cerebrovascular stenoses. AJNR Am J Neuroradiol 2005;26:538-42.

8. Derdeyn CP, Videen TO, Fritsch SM, et al. Compensatory mechanisms for chronic cerebral hypoperfusion in patients with carotid occlusion. Stroke 1999;30:1019-24.

9. Norrving B, Nilsson B, Risberg J. rCBF in patients with carotid occlusion. Resting and hypercapnic flow related to collateral pattern. Stroke 1982;13:155-62.

10. Baron JC, Bousser MG, Rey A, et al. Reversal of focal "misery-perfusion syndrome" by extraintracranial arterial bypass in hemodynamic cerebral ischemia. A case study with ${ }^{15} \mathrm{O}$ positron emission tomography. Stroke. 1981;12:454-59.

11. Cheung RT. The utility of melatonin in reducing cerebral damage resulting from ischemia and reperfusion. J Pineal Res 2003;34:153-60.

12. Sastry PS, Rao KS. Apoptosis and the nervous system. J Neurochem 2000;74:1-20.

13. Chen J, Jin K, Chen M, et al. Early detection of DNA strand breaks in the brain after transient focal ischemia:Implications for the role of DNA damage in apoptosis and neuronal cell death. J Neurochem 1997;69:232-45.

14. Pulsinelli WA, Brierley JB, Plum F. Temporal profile of neuronal damage in a model of transient forebrain ischemia. Ann Neurol. 1982;11:491-98.

15. Liem F, Mérillat S, Bezzola L, et al. Reliability and statistical power analysis of cortical and subcortical FreeSurfer metrics in a large sample of healthy elderly. Neuroimage 2015;108:95-109. 